Pacific Journal of Mathematics

A CLASS OF CONSISTENT ANTI-MARTIN'S AXIOMS 


\title{
A CLASS OF CONSISTENT ANTI-MARTIN'S AXIOMS
}

\author{
JOHN W. L. MERRILL
}

\begin{abstract}
Both the Continuum Hypothesis and Martin's Axiom allow inductive constructions to continue in circumstances where the inductive hypothesis might otherwise fail. The search for useful related axioms procedes naturally in two directions: towards "Super Martin's Axioms," which extend MA to broader classes of orders; and towards "Anti-Martin's Axioms" (AMA's) which are strictly weaker than $\mathrm{CH}$, but which, when combined with $\neg \mathrm{CH}$, deny MA. In this paper, we consider restrictions of van Douwen and Fleissner's Undefinable Forcing Axiom which are consistent with the negation of the continuum hypothesis.
\end{abstract}

1. Introduction. Baumgartner proposed an Anti-Martin's Axiom that he referred to as "The Complete Failure of Martin's Axiom," which asserts that for each c.c.c. order, there is a collection of $\omega_{1}$ dense subsets of that order which cannot be met by any filter. This axiom is clearly an AMA; it follows from $\mathrm{CH}$, is consistent with $\neg \mathrm{CH}$, and, in conjunction with the negation of $\mathrm{CH}$, implies the failure of MA. Unfortunately, a collection of essentially random subsets of a c.c.c. order yields only a very weak inductive capacity.

Another, less well-known, Anti-Martin's Axiom was introduced by van Douwen and Fleissner. Analyzing a model introduced by Bell and Kunen, they extracted an axiom they referred to as "The Definable Forcing Axiom," which captures some of the properties of that model. In this paper, we examine several other axioms closely related to DFA and capturing other facets of this model.

2. Notation and definitions. We assume that the reader is familiar with the terminology and notation of Martin's axiom and of iterated forcing. A good reference for the former is [9] or [5]; good references for the latter include [6] and [7].

All forcing-related axioms refer, however indirectly, to the notion of a generic filter through a partial order.

Definition 2.1. Let $\mathscr{M}$ be any model of ZFC, and let $\mathbf{P} \in \mathscr{M}$ be any partial order. $\mathscr{F}$ is an $\mathscr{M}$-generic filter over $\mathbf{P}$ if $\mathscr{F}$ is a filter in $\mathbf{P}$, and if, for each $\mathscr{D} \subset \mathbf{P}$ which is dense, $\mathscr{F} \cap \mathscr{D} \neq \varnothing$. 
For our purposes, the essential definition in [4] is that of an $\alpha$ generic sequence of filters. Since it is impossible for a non-trivial generic filter to exist, van Douwen and Fleissner examined sequences of filter which were cumulatively generic.

Definition 2.2. Let $\mathbf{P}$ be a partial ordering, and let $\alpha$ be an ordinal. An $\alpha$-generic sequence of filters over $\mathbf{P}$ is a sequence of filters, $\left\langle\mathscr{G}_{\beta}: \beta \in \alpha\right\rangle$, such that for each dense $\mathscr{D} \subset \mathbf{P}$, there is a $\xi \in \alpha$ such that for each $\beta \in(\xi, \alpha), \mathscr{D} \cap \mathscr{G}_{\beta} \neq \varnothing$.

In this paper, we will consider a still weaker version of genericity. Instead of studying the $\alpha$-genericity of sequences of filters, we will study the $\alpha$-genericity of sequences of centered families.

Definition 2.3. Let $\mathbf{P}$ be a partial order, and let $\mathscr{F} \subset \mathbf{P}$. Call $\mathscr{F}$ centered if and only if every finite subset of $\mathscr{F}$ has a lower bound in $\mathbf{P}$.

Definition 2.4. Let $\mathbf{P}$ be a partial ordering, and let $\alpha$ be an ordinal. An $\alpha$-generic sequence of centered families over $\mathbf{P}$ is a sequence of centered families, $\left\langle\mathscr{G}_{\beta}: \beta \in \alpha\right\rangle$, such that for each dense $\mathscr{D} \subset \mathbf{P}$, there is a $\xi \in \alpha$ such that for each $\beta \in(\xi, \alpha), \mathscr{D} \cap \mathscr{G}_{\beta} \neq \varnothing$.

A sequence of centered families is $\alpha$-generic if and only if every dense subset of the partial order in which they are embedded is eventually met by the members of the sequence. In this paper, when we refer to an $\alpha$-generic sequence, we shall mean an $\alpha$-generic sequence of centered families, not an $\alpha$-generic sequence of filters.

In [4], van Douwen and Fleissner propose the following axiom, there referred to as the Undefinable Forcing Axiom (UFA):

Axiom 2.5. Over each c.c.c. partial order of cardinality no greater than $\mathbf{c}$, there is an $\omega_{1}$-generic family of filters.

Todorčević observed that this axiom implies the continuum hypothesis. His proof, however, depends essentially upon the fact that the axiom guarantees the existence of a family of filters, and says nothing about the existence of families of centered families. Therefore, this paper studies a weaker form of UFA:

Axiom 2.6. Over each c.c.c. partial order of cardinality no greater than $\mathbf{c}$, there is an $\omega_{1}$ generic family of centered families.

Abusing notation, Axiom 2.6 will be referred to as UFA throughout this paper. This conflation of different axioms is justified by the circumstances in which they are applied. Either UFA is interesting only 
insofar as it applies to forcing constructions - and, when so restricted, the two axioms are equivalent. To show this, we need a somewhat non-standard definition.

Definition 2.7. A partial order $\mathbf{P}$ is well-met if and only if every compatible pair, $p \in \mathbf{P}$ and $q \in \mathbf{P}$, has a greatest lower bound.

LEMMA 2.8. If $\mathbf{P}$ is a well-met partial order, and $\mathscr{C}$ is any centered family contained in $\mathbf{P}$, then there is a filter $\mathscr{F} \supset \mathscr{C}$.

Proof. Set

$$
\mathscr{F}=\left\{p \in \mathbf{P}:(\exists k \in \omega)\left(\exists q_{1}, \ldots, q_{k} \in \mathscr{C}\right)\left(\bigcap_{i \leq k} q_{i}\right) \leq p\right\} .
$$

Since $\mathscr{C}$ is centered, every finite set of its elements has a meet. It is, therefore, evident that $\mathscr{F}$ consists of pairwise compatible elements, and that for any finite set of elements of $\mathscr{F}$, there is some element extending all of them. Moreover, $\mathscr{F}$ is closed upwards, and extends $\mathscr{C}$, as desired.

THEOREM 2.9. If $\mathbf{P}$ is a well-met partial order, then there is an $\omega_{1}$ generic sequence of filters over $\mathbf{P}$ if and only if there is an $\omega_{1}$-generic sequence of centered families over $\mathbf{P}$.

Proof. The forward implication is trivial-any $\omega_{1}$-generic sequence of filters is already an $\omega_{1}$-generic sequence of centered families.

To prove the reverse implication valid, let $\left\langle\mathscr{C}_{\alpha}: \alpha \in \omega_{1}\right\rangle$ be an $\omega_{1}$-generic sequence of centered families. Let $\left\langle\mathscr{F}_{\alpha}: \alpha \in \omega_{1}\right\rangle$ be a sequence of extensions of these families to filters. Observe that if any one of the centered families meets any fixed dense set, then so does the filter extending it; thus, $\left\langle\mathscr{F}_{\alpha}\right\rangle$ is an $\omega_{1}$-generic sequence of filters, as desired.

It is well known that every partial order embeds as a dense suborder of a well-met partial order of the same cardinality. (Just adjoin all formal meets of finite sets of compatible elements.) Moreover, it is known that most of the order-theoretic properties of the original partial order are inherited by the superstructure, and that among these are the countable chain condition, $\sigma$-centeredness, $(\sigma, k)$-linkedness for any $k$, and similar properties.

As a consequence of this inheritance, and of Theorem 2.9, Axiom 2.6 for all c.c.c. partial orders is equivalent to Axiom 2.5 for all wellmet partial orders, and the same equivalences hold for weakenings 
of these axioms. Since two orders are forcing-equivalent whenever they both embed densely in some larger order, it is acceptable to call Axiom 2.6 the Undefinable Forcing Axiom, since any application of Axiom 2.5 to construct a structure by internal forcing can be recast as an application of Axiom 2.6 to a well-met superorder of the original order.

In van Douwen and Fleissner's paper [4], the authors examined the properties of an axiom they refer to as DFA, which asserts the existence of $\omega_{1}$-generic sequences of filters for each c.c.c. partial order definable from a formula, and, possibly, a single real. Among the orders which can be defined in this fashion are the measure algebra and the Cohen order, for instance. There are, however, relatively wellbehaved orders which are not covered by this definition, such as the order which adds a new subset of $\omega$ which is almost contained in each element of a filter on $\omega$. These orders will be covered by the axioms discussed here.

\section{Definition 2.10. Let $\mathbf{P}$ be any partial order.}

- $F \subset \mathbf{P}$ is $k$-linked iff for each $M \subset F$ with $|M|=k$, there is a $q_{M} \in \mathbf{P}$ such that for each $p \in F, q_{M} \leq p$.

- A partial order is said to be $\sigma$-centered if it can be decomposed into the union of countably many centered families of elements.

- A partial order is $(\sigma, k)$-linked if it can be decomposed into the union of countably many $k$-linked families.

Observe that orders which are $\sigma$-centered or $(\sigma, k)$-linked for some $k$ are c.c.c. The purpose of this paper is to show that UFA is consistent with the negation of $\mathrm{CH}$ when it is restricted to such orders.

3. Consistency results. The Bell-Kunen model was originally constructed to prove the consistency of a certain property of the CechStone compactification of $\omega$. (That all points in the remainder $\omega^{*}$ have $\pi$-character $\omega_{1}$.) We shall use it to prove the consistency of several weak forms of UFA, all of which imply the same combinatorial result.

Definition 3.1. (The Bell-Kunen Model.) Let $\mathscr{M}$ be a model of ZFC. By induction on $\omega_{1}$, define a forcing extension of $\mathscr{M}$ by a c.c.c. order. For $\alpha=0$, let $\mathbf{P}_{0}=\varnothing$. For $\alpha \in \omega_{1}$, let $\dot{\mathbf{Q}}_{\alpha}$ be a $\mathbf{P}_{\alpha}$ name for an order such that $\mathbf{1} \vdash_{\mathbf{P}_{\alpha}}$ " $\dot{\mathbf{Q}}_{\alpha}$ is a c.c.c. order forcing MA and adding at least $\mathbf{c}^{+}$reals." Let $\mathbf{P}_{\alpha+1}=\mathbf{P}_{\alpha} * \dot{\mathbf{Q}}_{\alpha}$. For $\alpha$ a limit ordinal less than or equal to $\omega_{1}$, let $\mathbf{P}_{\alpha}$ be the direct limit of the sequence 
of preceding $\mathbf{P}_{\beta}$ 's. $\mathbf{P}_{\omega_{1}}$ is a Bell-Kunen order for $\mathscr{M}$. The sequence $\left\langle\left\langle\mathbf{P}_{\alpha} \mid \alpha \in \omega_{1}+1\right\rangle\left\langle\dot{\mathbf{Q}}_{\alpha} \mid \alpha \in \omega_{1}\right\rangle\right\rangle$ is a Bell-Kunen iteration for $\mathscr{M}$. If $G$ is $\mathbf{P}_{\omega_{1}}$-generic over $\mathscr{M}$, then $\mathscr{M}[G]$ is a Bell-Kunen model constructed over $\mathscr{M}$ (referred to as the Bell-Kunen model when the reference is clear).

Lemma 3.2, the essential technical lemma, states that if we are presented with a well-met partial order which can be "nicely" decomposed in the intermediate extensions, then the approximations in the intermediate stages will be "good enough" to generate an $\omega_{1}$-generic sequence of centered families of elements of the final order. The sets $X_{\alpha}$ are the universes of the approximations to the order. The ordinals $\xi_{\alpha}$ are the stages at which the decisions about the structure of the order close on themselves sufficiently that the centered families generated in the approximations are guaranteed to be "generic enough" in the final order.

Lemma 3.2. Let $\triangleleft$ be a $\mathbf{P}_{\omega_{1}}$-name and let $\gamma$ be an ordinal such that

$$
\mathbf{1} \Vdash “ \check{\gamma} \leq \mathbf{c}, "
$$

and

$$
\mathbf{1} \text { “ } \triangleleft \text { is a c.c.c. partial ordering of } \check{\gamma} \text {." }
$$

Also, let $\left\langle X_{\alpha}: \alpha \in \omega_{1}\right\rangle$ be a sequence of ground model sets, and let $\left\langle\xi_{\alpha}: \alpha \in \omega_{1}\right\rangle$ be a sequence of countable ordinals. Assume that for each $\alpha \in \omega_{1}$ and for each $\mathscr{M}$-generic filter over $\mathbf{P}, \mathscr{G}$, the following conditions hold in the extension $\mathscr{M}[\mathscr{G}]$ :

- $\left|X_{\alpha}\right|<\mathbf{c}$.

- $X_{\alpha} \subset X_{\alpha+1} \subset \gamma$.

- If $\alpha \in \omega_{1}$ and $F \in\left[X_{\alpha}\right]^{<\omega}$, then if

$$
(\exists p \in \mathscr{G}) p \Vdash \text { “ }(\exists \alpha \in \gamma)(\forall \xi \in F)(\alpha \triangleleft \xi) ”,
$$

then

$$
\left(\exists p \in \mathscr{G} \cap \mathbf{P}_{\xi_{\alpha}}\right) p \Vdash "(\exists \alpha \in \gamma)(\forall \xi \in F)(\alpha \triangleleft \xi) " .
$$

Then for any $\mathscr{H}$, an $\mathscr{M}$-generic filter over $\mathbf{P}_{\omega_{1}}, \mathscr{M}[\mathscr{H}]$ contains an $\omega_{1}$-generic sequence of centered families over the partial order $(\gamma, \triangleleft[\mathscr{H}])$

Proof. For each $\alpha \in \omega_{1}$, let

(6) $\sigma_{\alpha}=\left\{(p, \check{F}): p \in \mathbf{P}_{\omega_{1}}, \check{F} \in\left[X_{\alpha}\right]^{<\omega}\right.$, and

$$
p \Vdash “(\exists \alpha \in \gamma)(\forall \xi \in \check{F})(\alpha \triangleleft \xi) »\} .
$$


Observe first that $\sigma_{\alpha}$ is a $\mathbf{P}_{\omega_{1}}$-name which can be viewed as a $\mathbf{P}_{\xi_{\alpha}}$ name, by hypothesis. That is, considering the name

$$
\begin{aligned}
\tau_{\alpha}=\left\{(p, \check{F}): p \in \mathbf{P}_{\xi_{\alpha}}, \check{F} \in\left[X_{\alpha}\right]^{<\omega},\right. & \text { and } \\
& p \Vdash \text { “ “( }(\exists \alpha \in \gamma)(\forall \xi \in \check{F})(\alpha \triangleleft \xi) ”)\}
\end{aligned}
$$

one sees that if $\mathscr{H}$ is $\mathscr{M}$-generic over $\mathbf{P}$, then for each $F \in\left[X_{\alpha}\right]^{<\alpha}$,

$$
\left((\exists p \in \mathscr{H}) p \Vdash “ \check{F} \in \sigma_{\alpha} "\right) \Leftrightarrow\left(\left(\exists q \in\left(\mathscr{H} \cap \mathbf{P}_{\xi_{\alpha}}\right)\right) q \Vdash “ \check{F} \in \tau_{\alpha} "\right)
$$

Therefore, the set defined (in the final extension) by $\sigma_{\alpha}$ is already added by stage $\xi_{\alpha}$.

For the nonce, let us work in $\mathscr{M}[\mathscr{H}]$, where $\mathscr{H}$ is some arbitrary $\mathscr{M}$-generic filter over $\mathbf{P}_{\omega_{1}}$. Observe that the set $\sigma_{\alpha}[\mathscr{H}]$ is partially ordered by reverse extension. Moreover, observe also that the resulting partial order has the countable chain condition. Let $Y \in\left[\sigma_{\alpha}[\mathscr{H}]\right]^{\omega_{1}}$. For each $F \in Y$, let $\xi_{F}$ be such that for each $\zeta \in F, \xi_{F} \triangleleft \zeta$. (Note that the existence of such a $\xi_{F}$ is guaranteed by the membership of $F$ in $\sigma_{\alpha}$, but that the values of the individual minima may well not be computed at that stage. Thus, this selection must be performed in the final model.)

Since $(\gamma, \triangleleft[\mathscr{H}])$ is known to be c.c.c., there must exist finite sets $F \in Y$ and $G \in Y$ such that $\xi_{F}$ and $\xi_{G}$ are compatible relative to $\triangleleft[\mathscr{H}]$. By definition of $\sigma_{\alpha}$, then, $(F \cup G) \in \sigma_{\alpha}[\mathscr{H}], F \subset(F \cup G)$ and $G \subset(F \cup G)$, witnessing that $Y$ is not an antichain in $\sigma_{\alpha}[\mathscr{H}]$. Since possessing the countable chain condition is inherited downwards, $\sigma_{\alpha}$ is c.c.c. in all intermediate models in which its members are decided.

Moreover, since $\left|\sigma_{\alpha}\right|<\mathbf{c}$, there is some $\zeta_{\alpha}<\omega_{1}$ at which a $\mathscr{M}\left[\mathscr{H} \cap \mathbf{P}_{\zeta_{\alpha}}\right]$-generic subset is added to $\left(\sigma_{\alpha}, \supset\right)$. Denote that set by $\mathscr{H}_{\alpha}$. Let

$$
\mathscr{F}_{\alpha}=\left\{\beta \in \gamma:\left(\exists F \in \mathscr{H}_{\alpha}\right)(\forall \xi \in \gamma)[((\forall \zeta \in F)(\xi \triangleleft \zeta)) \Rightarrow(\xi \triangleleft \beta)]\right\},
$$

where the truth or falsehood of the expression in Equation (9) is computed in the final extension, not in the intermediate model.

These (the sequence $\left\langle\mathscr{F}_{\alpha}: \alpha \in \omega_{1}\right\rangle$ ) form an $\omega_{1}$-generic sequence for $(\gamma, \triangleleft[\mathscr{H}])$ in $\mathscr{M}[\mathscr{H}]$. To prove this, two facts must be demonstrated: first, that each $\mathscr{F}_{\alpha}$ is centered, and, second, that the sequence $\left\langle\mathscr{F}_{\alpha}\right\rangle$ is $\omega_{1}$-generic.

To show that each $\mathscr{F}_{\alpha}$ is centered, let $F \in\left[\mathscr{F}_{\alpha}\right]^{<\omega}$. For each $\beta \in$ $F$, let $F_{\beta} \in \mathscr{H}_{\alpha}$ witness the membership of $\beta$ in $\mathscr{F}_{\alpha}$. Since $\mathscr{H}_{\alpha}$ is a filter in $\left(\sigma_{\alpha}, \supseteq\right)$, and since that order is a well-met partial order, $\bigcup_{\beta \in F} F_{\beta} \in \sigma_{\alpha}$. By definition of $\sigma_{\alpha}$, then, there must be some element 
of $\gamma$ which extends each $\beta \in F$ relative to $\triangleleft$, proving that $\mathscr{F}_{\alpha}$ is centered.

To prove that $\left\langle\mathscr{F}_{\alpha}\right\rangle$ is $\omega_{1}$-generic, a certain correspondence must be exhibited: to each dense $\mathscr{D} \subset \gamma$ (dense in the partial ordering specified by $\triangleleft[\mathscr{H}])$ must be assigned an $\alpha_{\mathscr{D}} \in \omega_{1}$ such that for each $\beta>\alpha_{\mathscr{D}}, \mathscr{F}_{\alpha} \cap \mathscr{D} \neq \varnothing$.

Towards this end, let $\mathscr{D} \subset \gamma$ be dense in $\mathscr{M}[\mathscr{H}]$. Let

$$
A=\{\alpha \in \mathscr{D}:(\forall \beta \in \mathscr{D})(\alpha \neq \beta \Rightarrow \alpha \nless[\mathscr{H}] \beta)\} .
$$

Observe that $A$ is a maximal antichain in $(\gamma, \triangleleft[\mathscr{H}])$, and, therefore, is countable. Let $\delta \in \omega_{1}$ be large enough that $A \in \mathscr{M}\left[\mathscr{H} \cap \mathbf{P}_{\delta}\right]$ and $A \subset X_{\delta}$. Let $\alpha_{\mathscr{D}}=\max \left(\delta, \xi_{\delta}\right)$.

Let $\zeta \in \omega_{1}$ be an arbitrary ordinal such that $\xi_{\delta} \leq \zeta$. Since $A$ is an antichain in $(\gamma, \triangleleft[\mathscr{H}]), A^{\prime}=\{\{\alpha\}: \alpha \in A\}$ must be an antichain in $\left(\sigma_{\zeta}, \supset\right)$. Thus, $\mathscr{H}_{\zeta}$ (defined above) meets $A^{\prime}$; let $\{\beta\}$ be this intersection. (This set is a singleton because $A^{\prime}$ is an antichain.)

Since $\{\beta\} \in \mathscr{H}_{\zeta}, \beta$ is a member of $\mathscr{F}_{\zeta}$, by definition. By construction of $A^{\prime}, \beta \in A$; since $A \subset \mathscr{D}, \beta \in \mathscr{D}$.

Thus, for every $\zeta \geq \alpha_{\mathscr{D}}, \mathscr{F}_{\zeta} \cap \mathscr{D} \neq \varnothing$, and, therefore, $\left\langle\mathscr{F}_{\alpha}: \alpha \in\right.$ $\left.\omega_{1}\right\rangle$ is $\omega_{1}$-generic over $(\gamma, \triangleleft[\mathscr{H}])$, for any $\mathscr{M}$-generic set $\mathscr{H}$, as desired.

LEMMA 3.3. Let $k \in \omega$, and suppose that $\mathbf{P}$ can be decomposed into a countable union of $k$-linked families as

$$
\mathbf{P}=\bigcup_{n \in \omega} L_{n}^{k}
$$

Let $F$ be any non-empty subset of $\mathbf{P}$ with $|F| \leq k$. $F$ has a lower bound in $\mathbf{P}$ if and only if

$$
(\exists n \in \omega)(\forall p \in F)(\exists q \leq p)\left(q \in L_{n}^{k}\right) .
$$

Proof. $(\Rightarrow)$ Assume that $F$ has a lower bound in $\mathbf{P}$, and let $p$ be any such. For some $n, p \in L_{n}^{k}$, and that $L_{n}^{k}$ is a witness to the validity of (12).

$(\Leftarrow)$ By $(12)$, select an $n \in \omega$ and, for each $p \in F$, select a $q_{p} \leq p$ with $q_{p} \in L_{n}^{k}$. There are no more than $k$ different $q_{p}$ 's, and they all belong to a $k$-linked family, so they must have a common refinement in $\mathbf{P}$. That common refinement of $\left\{q_{p}: p \in F\right\}$ is also a common refinement of $F$ as was desired.

THEOREM 3.4. In the Bell-Kunen model, UFA holds for orders which are $(\sigma, k)$-linked for each $k$ in $\omega$. 
Proof. Let $\triangleleft$ be a $\mathbf{P}_{\omega_{1}}$-name for a partial order on some $\gamma \leq \mathbf{c}$ which is $(\sigma, k)$-linked for every $k$ in $\omega$. For each such $k$, let $\left\langle\lambda_{n}^{k}: n \in \omega\right\rangle$ be a sequence of names for subsets of $\gamma$ witnessing that $\triangleleft$ is $(\sigma, k)$ linked; that is, such that each $k \in \omega$,

$$
\mathbf{1} \Vdash “(\forall k \in \omega) \bigcup_{n \in \omega} \lambda_{n}^{k}=\gamma "
$$

and, moreover, such that for each $k \in \omega$ and each $n \in \omega$,

$$
\mathbf{1} \text { " } \lambda_{n}^{k} \text { is }(\sigma, k) \text {-linked." }
$$

As before, let us work in the extension for a short while. Let $\mathscr{H}$ be $\mathscr{M}$-generic over $\mathbf{P}_{\omega_{1}}$. We define a function, $f: \gamma \times \omega \times \omega \rightarrow 2$ by

$$
\text { (15) } f(\alpha, n, k)= \begin{cases}1 & \text { if there is some } \beta \in \gamma \text { such that } \beta \triangleleft[\mathscr{H}] \alpha \\ & \text { and } \beta \in \lambda_{n}^{k}[\mathscr{H}] \\ 0 & \text { otherwise. }\end{cases}
$$

Let $\phi$ be any $\mathbf{P}_{\omega_{1}}$-name for this function.

Since $\mathbf{P}_{\omega_{1}}$ is c.c.c., we can compute an a priori upper bound for how long it takes to decide the values of each of these names. Using this upper bound, let $\rho: \gamma \rightarrow \omega_{1}$ be a ground model function such that for each $\alpha \in \gamma$, each $k \in \omega$, each $n \in \omega$, and any $\mathscr{M}$-generic $\mathscr{G}$,

$$
((\exists p \in \mathscr{G}) p \| \phi(\alpha, n, k)) \Leftrightarrow\left(\left(\exists p \in\left(\mathscr{G} \cap \mathbf{P}_{\rho(\gamma)}\right)\right) p \| \phi(\alpha, n, k)\right) .
$$

Let $\left\langle\gamma_{\alpha}: \alpha \in \omega_{1}\right\rangle$ be an increasing sequence of ordinals strictly less than $\mathbf{c}$, the supremum of which is $\gamma$. For $\alpha \in \omega_{1}$, let

$$
X_{\alpha}=\left\{\beta \in \gamma_{\alpha}: \rho(\beta)<\alpha\right\} .
$$

Letting $\xi_{\alpha}=\alpha$ for each $\alpha \in \omega_{1}$, the sequences $\left\langle X_{\alpha}\right\rangle$ and $\left\langle\xi_{\alpha}\right\rangle$ are as required in the hypotheses of Lemma 3.2. Since $X_{\alpha}$ is a subset of $\gamma_{\alpha}$, the cardinality of $X_{\alpha}$ is always less than c. It is clear from the definition that for each $\alpha \in \omega_{1}, X_{\alpha} \subset X_{\alpha+1} \subset \gamma$. It only remains to show that comparability relative to $\triangleleft[\mathscr{H}]$ of elements of $X_{\alpha}$ is decided by elements of $\mathbf{P}_{\alpha}$.

By Lemma 3.3, groupwise comparability of some finite $F \subset X_{\alpha}$ is equivalent to the various members of $F$ having extensions belonging to some $\lambda_{n}^{k}[\mathscr{H}]$ for some $k \geq|F|$. By definition of $\phi$, this is equivalent to proving that for $n \in \omega$ and some $k \in \omega$ with $|F| \leq k$, there is some $p \in \mathscr{H}$ such that

$$
p \Vdash “(\forall \alpha \in \check{F})(\phi(\alpha, n, k)=1) " .
$$


It thus remains only to demonstrate that there is a $p \in \mathscr{H}$ satisfying (18) if and only if there is a $p \in\left(\mathscr{H} \cap \mathbf{P}_{\alpha}\right)$ satisfying (18). This is precisely the import of Equations (16) and (17).

Thus, $\left\langle X_{\alpha}: \alpha \in \omega_{1}\right\rangle$ satisfies the hypotheses of Lemma 3.2, and, therefore, UFA holds for $\triangleleft$.

We have the following immediate corollary.

Corollary 3.5. UFA ( $\sigma$-centered) holds in the Bell-Kunen model.

Merrill [8] has shown that UFA fails for orders of cardinality $\omega_{2}$ in the Bell-Kunen model. Lemma 3.2 shows that is the best possible result.

THEOREM 3.6. UFA holds for orders of cardinality $\omega_{1}$ in the BellKunen model.

Proof. Let $\triangleleft$ be a $\mathbf{P}_{\omega_{1}}$-name for a c.c.c. order on $\omega_{1}$. Let $X_{\alpha}=$ $\alpha+1$, and let $\xi_{\alpha}$ be any countable ordinal which is large enough to guarantee that for each finite $F \subset \alpha$, the compatibility or incompatibility of the members of $F$ is decided by stage $\xi_{\alpha}$. Applying Lemma 3.2 , we obtain the theorem.

4. Cardinal consequences of weak forms of UFA. In their paper, van Douwen and Fleissner [4] observed that UFA has interesting consequences contrasting strongly with those arising from MA. For instance, DFA implies that the reals are the union of $\omega_{1}$ null sets and of $\omega_{1}$ sets of first category. UFA, by contrast, implies that the continuum has cofinality $\omega_{1}$, and that for each uncountable $\kappa \leq \mathbf{c}, 2^{\kappa}=2^{\mathbf{c}}$. (Notice how different this is from the situation under MA.) In all but one of these cases, van Douwen and Fleissner's original proof goes through essentially unaltered using the conclusions of Theorem 3.4 instead of either DFA or UFA.

The proof that the reals are the union of $\omega_{1}$ sets of first category if the conclusions of Theorem 3.4 hold consists mostly of observing that measure algebra is $(\sigma, k)$-linked for each $k$, and that an $\omega_{1}$ generic sequence through the measure algebra adds an $\omega_{1}$ sequence of first-category sets which cover the real line. Similarly, the proof that the reals are the union of $\omega_{1}$ null sets in any model in which the conclusions of Theorem 3.4 hold consists of the same argument, with "Cohen algebra" substituted for "measure algebra" and "null set" for "set of first category". The only differences between van Douwen 
and Fleissner's proof and ours is that it is often easier to show that an order is $(\sigma, k)$-linked than it is to show that it is real-definable.

Similarly, van Douwen and Fleissner's characterization of the continuum function under UFA also follows from the conclusions of Theorem 3.4, or even the conclusion of the weaker Corollary 3.5. One of their two proofs goes through completely unaltered; the other requires some work. To demonstrate that the confinality of $\mathbf{c}$ is $\omega_{1}$, for instance, van Douwen and Fleissner observe that every non-principal ultrafilter on $\omega$ has small $\pi$-character if UFA holds, and then invoke a result of Kunen's to prove that $c f(\mathbf{c})=\omega_{1}$. That proof goes through unaltered using UFA ( $\sigma$-centered) instead of full UFA.

One of their proofs does not quite go through unaltered. The proof of Lemma 4.2 presented in [4] employs an order which is not obviously $(\sigma, k)$-linked for each $k$. For completeness, therefore, the following version of their proof is presented here. This version makes use of an order which is not only $(\sigma, k)$-linked for each $k$, but even $\sigma$-centered.

We shall require the following definition:

Definition 4.1 The $\omega_{1}$-Borel sets over a topological space $\mathscr{T}$ are the members of the least family of subsets of that space containing all the members of $\mathscr{O}(\mathscr{T})$ and closed under complement and union or intersection of subfamilies of cardinality no more than $\omega_{1}$.

Lemma 4.2. Assume the conclusions of Theorem 3.4. Then every subset of the reals is an $\omega_{1}$-Borel set.

Proof. Let $X$ be a subset of $\mathbf{R}$. Let $\mathbf{P}_{X}$ be a $\sigma$-centered order making $X$ a $G_{\delta}$ in the extension, i.e.

$$
\begin{aligned}
\mathbf{P}_{X}=\{ & \left\langle\mathscr{U}_{i}, X_{i}\right\rangle_{i<k}: k<\omega, \\
& \text { each } \mathscr{U}_{i} \text { is a finite union of open, rational intervals, } \\
& \left.X_{i} \in[\mathbf{R} \backslash X]^{<\omega}, \text { and }(\forall i<n)\left(\mathscr{U}_{i} \cap\left(\bigcup_{j \leq i} X_{j}\right)\right)=\varnothing\right\}
\end{aligned}
$$

ordered by reverse inclusion on the common factors:

$$
\left\langle\mathscr{U}_{i}, X_{i}\right\rangle_{i<k} \triangleleft\left\langle\mathscr{V}_{j}, Y_{j}\right\rangle_{j<l}
$$


if and only if

(1) $k \geq l$,

(2) for each $j<l, \mathscr{U}_{j} \supseteq \mathscr{V}_{j}$, and

(3) for each $j<l, X_{j} \supseteq Y_{j}$.

Observe that $\mathbf{P}_{X}$ is $\sigma$-centered, and that

$$
\begin{aligned}
& \mathbf{1} \Vdash \text { “ } X=\check{\mathbf{R}} \cap\left(\bigcap _ { i \in \omega } \cup \left\{\mathscr{U}_{i}:(\exists p \in \Gamma)\right.\right. \\
& \left.\left.\quad\left(p=\left\langle\mathscr{U}_{i} \cdots \mathscr{U}_{i-1} \mathscr{U}_{i} \cdots ; \vec{X}\right\rangle\right)\right\}\right) . "
\end{aligned}
$$

Let $\left\langle\mathscr{G}_{\alpha}: \alpha \in \omega_{1}\right\rangle$ be an $\omega_{1}$-generic sequence for $\mathbf{P}_{X}$. For $\alpha \in \omega_{1}$, let $G_{\alpha}=\left(\bigcap_{i \in \omega} \cup\left\{\mathscr{U}_{i}:\left(\exists p \in \mathscr{G}_{\alpha}\right)\left(p=\left\langle\mathscr{U}_{i} \cdots \mathscr{U}_{i-1} \mathscr{U}_{i} \cdots ; \vec{X}\right\rangle\right)\right\}\right)$.

Claim. $X=\bigcup_{\alpha \in \omega_{1}}\left(\bigcap_{\beta=\alpha}^{\omega_{1}} G_{\beta}\right)$.

If $y \notin X$, then there is a tail of the filters hitting the dense set of conditions forcing that $(\exists j \in \omega) y \notin X_{j}$; thus, for each $\alpha \in \omega_{1}$, $\cap_{\beta=\alpha}^{\omega_{1}} G_{\beta}$ is a subset of $X$. If $x \in X$, then there is a tail of the filters which, for each $n$, hit the dense set which says $(\exists p \in \Gamma)\left(x \in \mathscr{U}_{n}^{p}\right)$, or, equivalently, there is a tail of filters such that $x \in G_{\alpha}$.

Corollary 4.3 (van Douwen and Fleissner, see [4]). Assume UFA for $\sigma$-centered partial orders. Then $2^{\mathbf{c}}=2^{\omega_{1}}$.

Proof. There are $2^{\mathrm{c}}$ subsets of the reals, and only $2^{\omega_{1}} \omega_{1}$-Borel sets. Thus, $2^{\omega_{1}} \geq 2^{\mathbf{c}}$; of course, $2^{\mathbf{c}} \geq 2^{\omega_{1}}$.

5. Conclusions and open questions. Kunen (pers. comm.) has characterized the structure of the unsplit Hausdorff gaps in the Bell-Kunen model.

THEOREM 5.1 (Kunen). In the Bell-Kunen model, every unsplit Hausdorff gap is a $\left(\kappa, \omega_{1}\right)$-gap for some $\kappa<\mathbf{c}$.

It can be easily shown that this result follows from Lemma 3.2, because there is an order which splits an unsplit Hausdorff gap the members of which consist of finite sets of members of the gap, order thereon being decided purely by the elements of the members of the gap.

Baumgartner [2] points out that this order has precaliber $\omega_{1}$ in every case where Theorem 5.1 applies, and, therefore a more direct 
proof of the theorem would follow from UFA restricted to orders with precaliber $\omega_{1}$. The present author has been unable to find either a proof or a refutation of this axiom in the Bell-Kunen model. It would, therefore, by very interesting to resolve the truth or falsehood of the following assertion:

Conjecture 5.2. In the Bell-Kunen model, UFA holds for all c.c.c. partial orders with precaliber $\omega_{1}$. (Or, in fact, for all c.c.c. partial orders with property $K$.)

We have presented several restrictions of UFA which are consistent with the negation of the continuum hypothesis, and we have shown that they have non-obvious consequences for the structure of the continuum. Along with the truth or falsehood of Conjecture 5.2, it would be interesting to know the answers to any of the following open questions.

1. Is UFA restricted to orders of cardinality $\omega_{2}$ consistent with the negation of the continuum hypothesis?

2. Is the negation of $\mathrm{CH}$ consistent UFA for c.c.c.-productive orders?

3. Is UFA restricted to $\sigma$-linked orders consistent with $2^{\omega}>\omega_{1}$ ?

\section{REFERENCES}

[1] J. Baumgartner, The absolute failure of Martin's axiom, (preprint).

[2] _ The proper forcing axiom, The Handbook of Set-theoretic Topology (K. Kunen and J. Vaugn, eds., eds.), North-Holland, New York, 1986.

[3] M. Bell and K. Kunen, On the Pi-character of ultrafilters, C.R. Math. Rep. Acad. Sci. Canada, III (1981), 351-346.

[4] E. K. van Douwen and W. Fleissner, The definable forcing axiom, (preprint).

[5] D. Fredmlin, Martin's Axiom, Cambridge Tracts on Mathematics, Cambridge University Press, Cambridge, 1986.

[6] T. Jeck, Set Theory, Academic Press, New York, 1980.

[7] K. Kunen, Set Theory, North-Holland, New York, 1980.

[8] J. W. L. Merrill, UFA fails in the Bell-Kunen Model, J. Symbolic Logic, (in press).

[9] M. E. Rudin, Martin's Axiom, The Handbook of Mathematical Logic (J. Barwise, ed., eds.), North-Holland, New York, 1979.

Received January 11, 1988 and in revised form June 21, 1989. This work is taken from the author's doctoral dissertation. Preparation of this paper was supported partly by AFOSR Contract Number F49620-86-C0037. 


\section{PACIFIC JOURNAL OF MATHEMATICS EDITORS}

\author{
V. S. VARADARAJAN \\ (Managing Editor) \\ University of California \\ Los Angeles, CA 90024-1555-05 \\ Herbert Clemens \\ University of Utah \\ Salt Lake City, UT 84112 \\ ThOMAs ENRIGHT \\ University of California, San Diego \\ La Jolla, CA 92093
}

R. FINN

Stanford University

Stanford, CA 94305

HeRmanN FlaschKa

University of Arizona

Tucson, AZ 85721

VAUGHAN F. R. JoNES

University of California

Berkeley, CA 94720

SteVen KerCKhofF

Stanford University

Stanford, CA 94305
ROBION KIRBY

University of California

Berkeley, CA 94720

C. C. Moore

University of California

Berkeley, CA 94720

HAROLD STARK

University of California, San Diego

La Jolla, CA 92093

\section{ASSOCIATE EDITORS}
R. ARENS
E. F. BECKENBACH
B. H. NeumanN
F. Wolf
K. YoshidA
(1906-1982)
(1904-1989)

\section{SUPPORTING INSTITUTIONS}

UNIVERSITY OF ARIZONA
UNIVERSITY OF BRITISH COLUMBIA
CALIFORNIA INSTITUTE OF TECHNOLOGY
UNIVERSITY OF CALIFORNIA
MONTANA STATE UNIVERSITY
UNIVERSITY OF NEVADA, RENO
NEW MEXICO STATE UNIVERSITY
OREGON STATE UNIVERSITY
UNIVERSITY OF ARIZONA
UNIVERSITY OF BRITISH COLUMBIA
UNIVERSITY OF CALIFORNIA
MONTANA STATE UNIVERSITY
NEW MEXICO STATE UNIVERSITY

\author{
UNIVERSITY OF OREGON \\ UNIVERSITY OF SOUTHERN CALIFORNIA \\ STANFORD UNIVERSITY \\ UNIVERSITY OF HAWAII \\ UNIVERSITY OF TOKYO \\ UNIVERSITY OF UTAH \\ WASHINGTON STATE UNIVERSITY \\ UNIVERSITY OF WASHINGTON
}

The Supporting Institutions listed above contribute to the cost of publication of this Journal, but they are not owners or publishers and have no responsibility for its content or policies.

Mathematical papers intended for publication in the Pacific Journal of Mathematics should be in typed form or offset-reproduced (not dittoed), double spaced with large margins. Please do not use built up fractions in the text of the manuscript. However, you may use them in the displayed equations. Underline Greek letters in red, German in green, and script in blue. The first paragraph must be capable of being used separately as a synopsis of the entire paper. In particular it should contain no bibliographic references. Please propose a heading for the odd numbered pages of less than 35 characters. Manuscripts, in triplicate, may be sent to any one of the editors. Please classify according to the 1980 Mathematics Subject Classification (1985 Revision) scheme which can be found in the December index volumes of Mathematical Reviews. Supply name and address of author to whom proofs should be sent. All other communications should be addressed to the managing editor, or Elaine Barth, University of California, Los Angeles, California 90024-1555-05.

There are page-charges associated with articles appearing in the Pacific Journal of Mathematics. These charges are expected to be paid by the author's University, Government Agency or Company. If the author or authors do not have access to such Institutional support these charges are waived. Single authors will receive 50 free reprints; joint authors will receive a total of 100 free reprints. Additional copies may be obtained at cost in multiples of 50 .

The Pacific Journal of Mathematics is issued monthly as of January 1966. Regular subscription rate: $\$ 190.00$ a year (6 Vols., 12 issues). Special rate: $\$ 95.00$ a year to individual members of supporting institutions.

Subscriptions, orders for numbers issued in the last three calendar years, and changes of address should be sent to Pacific Journal of Mathematics, P.O. Box 969, Carmel Valley, CA 93924, U.S.A. Old back numbers obtainable from Kraus Periodicals Co., Route 100, Millwood, NY 10546.

The Pacific Journal of Mathematics at P.O. Box 969, Carmel Valley, CA 93924 (ISSN 0030-8730) is published monthly. Second-class postage paid at Carmel Valley, California 93924, and additional mailing offices. Postmaster: send address changes to Pacific Journal of Mathematics, P.O. Box 969, Carmel Valley, CA 93924.

\section{PUBLISHED BY PACIFIC JOURNAL OF MATHEMATICS, A NON-PROFIT CORPORATION}




\section{Pacific Journal of Mathematics}

\section{Vol. 143, No. $2 \quad$ April, 1990}

Gustavo Corach, Horacio Porta and Lázaro Recht, Differential geometry of systems of projections in Banach algebras ................. 209

Peter Fleischmann and Jens Carsten Jantzen, Simple periodic modules of

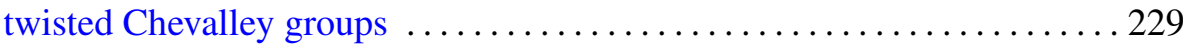

Niels Gronbaek, Amenability of discrete convolution algebras, the commutative case ...................................243

Nguyên H. V. Hung, The mod 2 equivariant cohomology algebras of

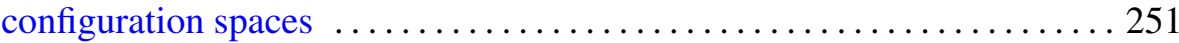

Wojciech Kucharz, Global almost analytic algebraicity of analytic sets . . . 287 John Merrill, A class of consistent anti-Martin's axioms .............. 301

Takafumi Murai, The power 3/2 appearing in the estimate of analytic

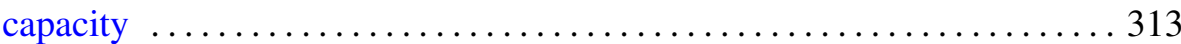

L. Panaitopol and Doru Stefanescu, On the generalized difference

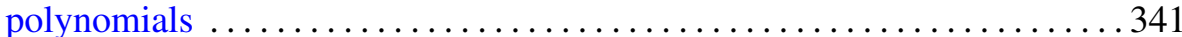

Katsuhiro Shiohama, Takashi Shioya and Minoru Tanaka, Mass of rays

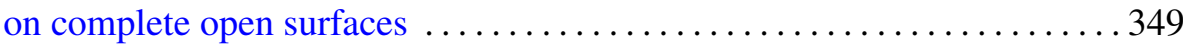

Gerhard Ströhmer, About compressible viscous fluid flow in a bounded

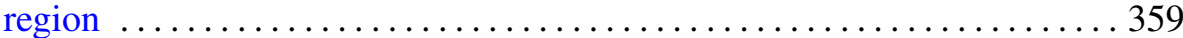

A. Ülger, Arens regularity sometimes implies the RNP 